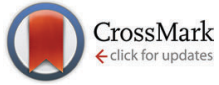

Cite this: Soft Matter, 2015, 11, 4295

Received 31st March 2015, Accepted 7th April 2015

DOI: $10.1039 / \mathrm{c} 5 \mathrm{sm} 00760 \mathrm{~g}$

www.rsc.org/softmatter

\title{
Helical inner-wall texture prevents jamming in granular pipe flows
}

\author{
Felix Verbücheln, Eric J. R. Parteli and Thorsten Pöschel*
}

\begin{abstract}
Granular pipe flows are characterized by intermittent behavior and large, potentially destructive solid fraction variations in the transport direction. By means of particle-based numerical simulations of gravity-driven flows in vertical pipes, we show that it is possible to obtain steady material transport by adding a helical texture to the inner-wall of the pipe. The helical texture leads to a more homogeneous mass flux along the pipe, prevents the emergence of large density waves and substantially reduces the probability of plug formation thus avoiding jamming of the particulate flow. We show that the granular mass flux $Q$ through a pipe of diameter $D$ with a helical texture of wavelength $\lambda$ follows the equation $Q=Q_{0} \cdot\{1-B \sin [\arctan (2 \pi D / \lambda)]\}$, where $Q_{0}$ is the flow without helix, predicted from the well-known Beverloo equation. Our new expression yields, thus, a modification of the Beverloo equation with only one additional fit parameter, $B$, and describes the particle mass flux with the helical texture with excellent quantitative agreement with simulation results. Future application of the method proposed here has the potential to improve granular pipe flows in a broad range of processes without the need for energy input from any external source.
\end{abstract}

\section{Introduction}

The transport of a granular material flowing through a pipe is a process of relevance for a broad range of scientific and technological areas. ${ }^{1,2}$ One fundamental problem in such transport is that the material flow is intrinsically unstable and characterized by large variations in the solid fraction (density waves) along the pipe. ${ }^{3,4}$ These waves induce potentially destructive pressure transients on the inner-wall of the pipe and provide the mechanism responsible for the intermittent behavior of the flow.

Although the dynamics of density waves in granular pipe flows have been studied extensively in the past both experimentally $^{5-10}$ and through different types of models, ${ }^{3,4,11-19}$ it is still a challenging problem to control the mass flux of the granular material flowing through a pipe. For example, Zuriguel $e t a l^{20}$ showed how insertion of an obstacle just above the outlet of a silo can significantly reduce the probability that the granular flow is arrested due to the formation of an arch blocking the silo's outlet. ${ }^{20}$ However, most of the proposed strategies to control the transport along the pipe involve energy input from an external source, e.g. through application of electric fields ${ }^{21}$ or mechanical perturbations. ${ }^{1,22}$

Institute for Multiscale Simulation, Friedrich-Alexander University of Erlangen-Nuremberg, Nägelsbachstraße 49b, 91052 Erlangen, Germany.

E-mail: thorsten.poeschel@fau.de; Tel: +4991318520867
Here we demonstrate a method to homogenize the mass flux and avoid flow blockage in granular pipe flows without the necessity of applying any external source of energy to the system. Our method consists of adding to the inner-wall of the pipe a helical texture, which leads to a more homogeneous distribution of the particles within the pipe thus avoiding the formation of stable plugs. By means of particle-based numerical experiments, we will show that it is possible to achieve flows with prescribed characteristics regarding the particle distribution within the pipe and the mass flow rate of the granular material by adjusting the geometric properties of the helix-shaped texture.

\section{Numerical experiments}

We simulate the process using the Discrete Element Method (DEM), that is, simultaneously solving Newton's equations of translational and rotational motion for all particles. There is a variety of models to describe the contact forces in DEM simulations, which are suitable for different particle geometry and material behavior. ${ }^{23-26}$ In the present paper, we assume viscoelastic interaction in the normal direction ${ }^{27}$ and apply a modified Cundall-Strack model ${ }^{28}$ for the tangential direction. ${ }^{29}$ The corresponding forces read

$$
\vec{F}_{n}=\min \left(0,-\rho \xi^{3 / 2}-\frac{3}{2} A_{n} \rho \sqrt{\xi} \dot{\xi}\right) \vec{e}_{n},
$$

where

$$
\xi=R_{1}+R_{2}-\left|\vec{r}_{1}-\vec{r}_{2}\right|
$$


is the compression of particles of radii $R_{1}$ and $R_{2}$ at positions $\vec{r}_{1}$ and $\vec{r}_{2}$, and $\vec{e}_{n} \equiv\left(\vec{r}_{1}-\vec{r}_{2}\right) /\left|\vec{r}_{1}-\vec{r}_{2}\right|$ is the normal unit vector. The elastic parameter of eqn (1), $\rho$, is a function of the Young's modulus, $Y$, the Poisson's ratio $\nu$, and the effective radius $R_{\text {eff }} \equiv R_{1} R_{2} /\left(R_{1}+R_{2}\right)$,

$$
\rho \equiv \frac{2 Y}{3\left(1-\nu^{2}\right)} \sqrt{R_{\mathrm{eff}}},
$$

while the dissipative parameter, $A_{n}$, further depends on the material viscosities. ${ }^{27}$ While $\rho$ can be computed directly from material properties which are easily available for a variety of materials, the viscosities needed for $A_{n}$ are not directly available. To determine $A_{n}$, therefore, we use a relation between the coefficient of restitution, $\varepsilon$, for the collision of two isolated particles, the pre-collisional velocity of these particles, $v_{\text {imp }}$, and $A_{n},{ }^{30-32}$ where the Padé approximation is employed as described elsewhere. ${ }^{33}$

The tangential force reads ${ }^{29}$

$$
\vec{F}_{t}=-\min \left[\mu\left|\vec{F}_{n}\right|, \int_{\mathrm{path}} \frac{4 G}{2-\nu} \sqrt{R_{\mathrm{eff}} \xi} \mathrm{d} s+A_{t} \sqrt{R_{\mathrm{eff}} \xi} v_{t}\right] \vec{e}_{t},
$$

where $\mu$ is the Coulomb friction coefficient and $G$ is the shear modulus, which is given by the equation, $2 G=Y /(1+\nu)$. The integral in eqn (4) is performed over the displacement of the particles at the point of contact for the duration of the contact. ${ }^{28}$ Moreover, $\vec{v}_{t}=v_{t} \vec{e}_{t}$ stands for the relative tangential velocity at the point of contact, where $\vec{e}_{t}$ is the corresponding unit vector. The tangential dissipative parameter, $A_{t}$, characterizes the surface roughness and is chosen such that the prefactors of the normal and tangential deformation rates $\left(\dot{\xi}\right.$ and $v_{t}$ ) in eqn (1) and (4), respectively, are of the same order of magnitude. ${ }^{34}$ Using this assumption, previous authors ${ }^{35}$ found excellent agreement between simulation results and experimental values of particle velocity profiles in a gravitydriven shearing experiment. By comparing eqn (1) and (4), we obtain $A_{t} \approx A_{n} Y /\left(1-\nu^{2}\right)$.

The integration was performed using LIGGGHTS, ${ }^{29}$ and the values of the model parameters are listed in Table 1 . The equations used for computing the forces between particles and the internal (frictional) wall of the cylinder are the same as that used for modeling particle-particle collisions where one of the contact partners is of infinite mass and radius. Moreover, in order to compute the viscoelastic constant $A_{n}$ using an analytical model, ${ }^{33}$ we assume a coefficient of restitution $\varepsilon \approx 0.5$ associated with a pre-collisional velocity $v_{\text {imp }} \approx 1.0 \mathrm{~m} \mathrm{~s}^{-1}$, which is of the same order of the average axial particle velocities found in

Table 1 Numerical values of the parameters used in the simulations

\begin{tabular}{lll}
\hline Parameter & Symbol & Value \\
\hline Particle material density & $\rho_{\mathrm{p}}$ & $2650 \mathrm{~kg} \mathrm{~m}^{-3}$ \\
Particle diameter & $d$ & $1.2 \mathrm{~mm}$ \\
Young's modulus & $Y$ & $10^{8} \mathrm{~Pa}$ \\
Poisson's ratio & $\nu$ & 0.24 \\
Coulomb's friction coefficient & $\mu$ & 0.5 \\
Pipe length & $L$ & $1 \mathrm{~m}$ \\
Timestep & $\Delta t$ & $6 \times 10^{-7} \mathrm{~s}$
\end{tabular}

our simulations as discussed below. Using these parameters and the material properties specified in Table 1 , we obtain $A_{n} \approx$ $7.3 \times 10^{-6}$ and $A_{t} \approx 775.9$ for particle-particle collisions, while for particle-wall collisions these values are $A_{n} \approx 8.4 \times 10^{-6}$ and $A_{t} \approx 891.3$.

The integration time step $\Delta t$ must be small enough to accurately solve Newton's equations for the particle interaction. For undamped collisions, the duration $T_{\text {col }}$ of the collision can be estimated using the equation, ${ }^{23}$

$$
T_{\mathrm{col}} \approx 3.21\left(M_{\mathrm{eff}} / \rho\right)^{2 / 5} \cdot v_{\mathrm{imp}}^{-1 / 5},
$$

where $M_{\text {eff }}=m_{1} m_{2} /\left(m_{1}+m_{2}\right)$ with $m_{1}$ and $m_{2}$ standing for the masses of the interacting particles. Typically a timestep smaller than about $T_{\text {col }} / 50$ is recommended. ${ }^{36}$ Since the collision time $T_{\text {col }}$ computed using eqn (5) with the material properties specified above is about $50 \mu \mathrm{s}$, we use here $\Delta t \approx 6 \times 10^{-7} \mathrm{~s}$, which is below the recommended upper bound for $\Delta t$ mentioned above.

The pipe has a circular cross-section of diameter $D \mathrm{~mm}$ and its length is $L=1 \mathrm{~m}$. At time $t=0, N$ particles are placed at random positions within the pipe, where $N$ is chosen such that the sum of the volumes of all particles amounts to a prescribed fraction $V_{\phi}$ of the pipe volume. The initial velocity of the particles in the radial direction is chosen randomly between $-v_{\mathrm{r}}$ and $v_{\mathrm{r}}$, with $v_{\mathrm{r}}=0.01 \mathrm{~m} \mathrm{~s}^{-1}$, while the particles have vanishing initial velocity in the axial direction $\left(v_{z}=0\right)$. Periodic boundary conditions are applied in the vertical $(z)$ direction. We have performed simulations with pipes longer than $L=1 \mathrm{~m}$ and found that the results presented in the next section with this value of $L$ are not affected by finite size effects. That is, increasing the length of the pipe does not change the results.

\section{Results and discussion}

We performed simulations using a constant particle diameter $d$ as specified in Table 1 and different values of pipe diameter $D$. We found that the flow behavior depends fundamentally on the pipe to particle diameter ratio $D_{\phi} \equiv D / d$. For $D_{\phi}>3$ the flow is intermittent and characterized by a clogging regime where the average particle velocity in the vertical direction is nearly constant (Section 3.1). For $D_{\phi} \lesssim 3$ jamming occurs thus leading to blockage of the granular flow (Section 3.2). Following the discussion of these distinct flow regimes we show in Section 3.3 that adding a helical inner-wall texture leads to a more homogeneous solid fraction distribution along the pipe thus reducing the occurrence of clogging and preventing the flow to jam.

\subsection{Clogging regime}

Fig. 1a shows the evolution of the total kinetic energy of particles flowing through a vertical pipe with $D_{\phi}=4$. Since the initial particle velocities in the radial direction are small, the particles fall freely under the action of gravity during an initial time before colliding with the inner wall of the pipe. Collisions between the particles and between the particles and the wall lead, then, to deceleration of the particles and a 

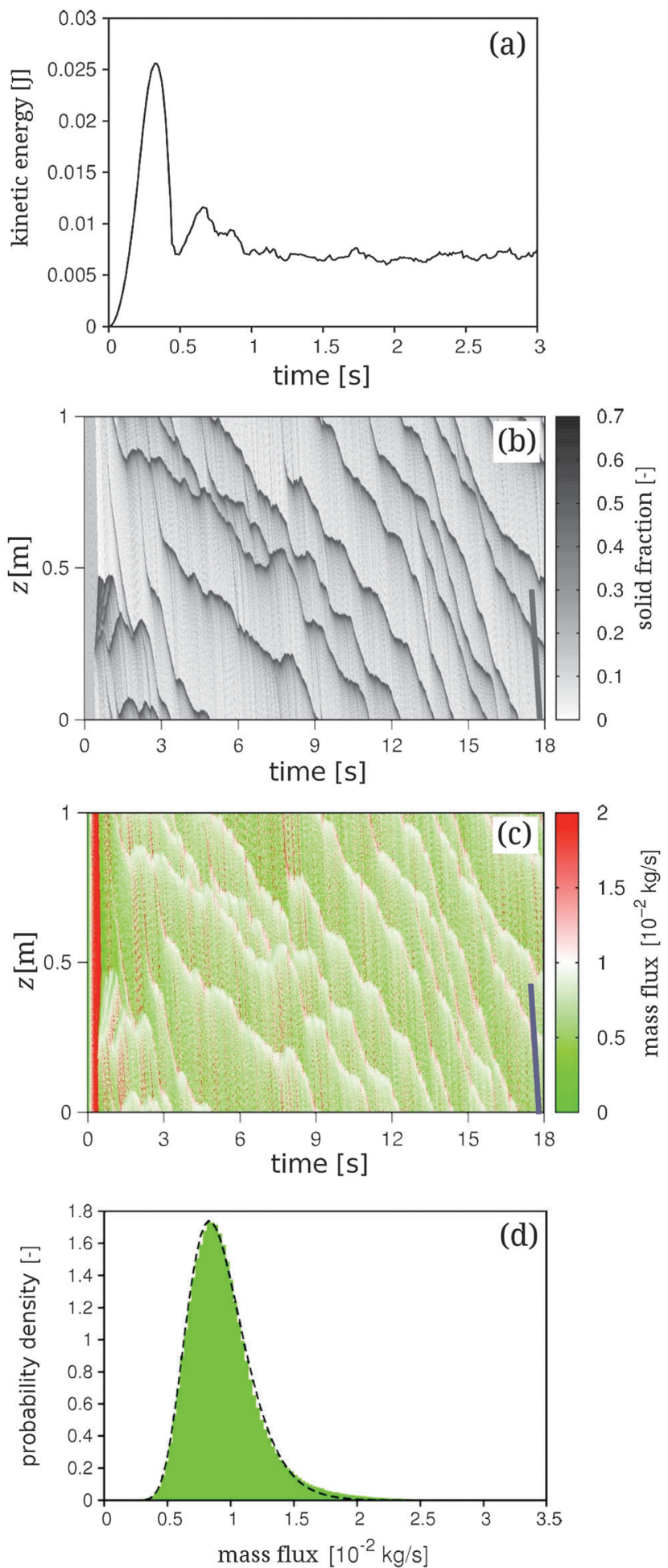

Fig. 1 Granular flow through a pipe with $D_{\phi}=4$ (clogging regime). (a) Total kinetic energy of the particles as a function of time; (b) and (c) spatio-temporal images of the packing fraction and mass flux, respectively, along the tube; (d) density distribution of the time-averaged mass flow. The slope of the line at the lower right corner indicates the average axial velocity of the particles.

decrease in the kinetic energy of the system. After a transient time, the energy gain of the particles due to gravity is nearly compensated by the energy dissipation due to collisions such that the total kinetic energy fluctuates around a constant value. The spatio-temporal image of the volume fraction along the tube (Fig. 1b) shows the emergence of density waves, ${ }^{3}$ with the development of recurrent clogging characterized by the formation of plugs, that is regions with a high packing fraction (thick dark lines in Fig. 1b) which can either converge or diverge with time. The flow is inhomogeneous and associated with strong fluctuations of particle average velocities and solid fraction both in time and position along the pipe.

Fig. 1c shows the spatio-temporal image of the areaintegrated particle mass flux per unit time along the tube. This flux is defined as,

$$
\dot{m}(t, z)=\rho_{\mathrm{p}} \varphi(t, z)\left[v_{z}(t, z)\right] \pi D^{2} / 4,
$$

where $\varphi(t, z)$ and $v_{z}(t, z)$ are the packing fraction and the average particle velocity in the vertical direction, respectively, at time $t$ within the volume element $[z+\mathrm{d} z] \pi D^{2} / 4$. The probability density distribution $f\left(\dot{m}_{0}\right)$ of the mass flux $\dot{m}_{0} \equiv \dot{m}(t, 0)$ at the bottom of the pipe, that is at $z=0$, is shown in Fig. 1 d. We see that $f\left(\dot{m}_{0}\right)$ can be well described by a lognormal distribution (fit represented by the dashed line in Fig. 1d). The expectation value of this distribution is around $0.0092 \mathrm{~kg} \mathrm{~s}^{-1}$ and nearly equals, thus, the value of mass flux associated with plug flow, denoted by the thick bright meandering lines in Fig. 1c.

Moreover, the thinner lines in the spatio-temporal plot of Fig. 1c denote faster moving particle groups and are associated with different solid fractions, as can be seen from Fig. $1 \mathrm{~b}$. Indeed, these lines denote smaller particle groups which occur in-between the plugs during the intermittent flow. Since these thinner lines align nearly parallel to each other, we conclude that the small interplug particle groups all move with nearly the same average axial velocity down the pipe, independently of the value of the solid fraction. Among these smaller groups, the ones with the lowest (highest) values of packing fraction are associated with the smallest (largest) values of mass flux-that is to the thin green (red) lines-in the spatio-temporal diagram of Fig. 1c. In other words, the left (right) tail of the probability density distribution in Fig. 1d incorporates the flux due to the small inter-plug particle groups with the lowest (highest) solid fractions, whereas it is the flux due to plug flow that dictates the expectation value of this distribution.

We have calculated $f\left(\dot{m}_{0}\right)$ for different values of $D_{\phi}$ within the range between 3.5 and 7.0. Fig. 2a shows the respective distributions. We see that both the distribution width and expectation value increase with the diameter ratio $D_{\phi}$. Indeed, for a given particle size and volume fraction, both the particle number and average axial velocity-and thus also the average mass flux-increase with $D_{\phi}$. Also the distance between plugs increases with the diameter ratio $D_{\phi}$, which means that the small inter-plug particle groups can accelerate the longer the larger the pipe diameter.

Moreover, in Fig. 2b we see the time-averaged flux, that is the expectation value $Q_{0}=\left\langle\dot{m}_{0}\right\rangle$, as a function of the pipe diameter $D$ as obtained from the simulations (circles). Note that this mean flux can be written as $Q_{0}=j A$, where $A=\pi D^{2} / 4$ is the area of the pipe cross-section and $j=\left\langle v_{z}\right\rangle \rho_{\mathrm{p}} V_{\phi}$ is the average 

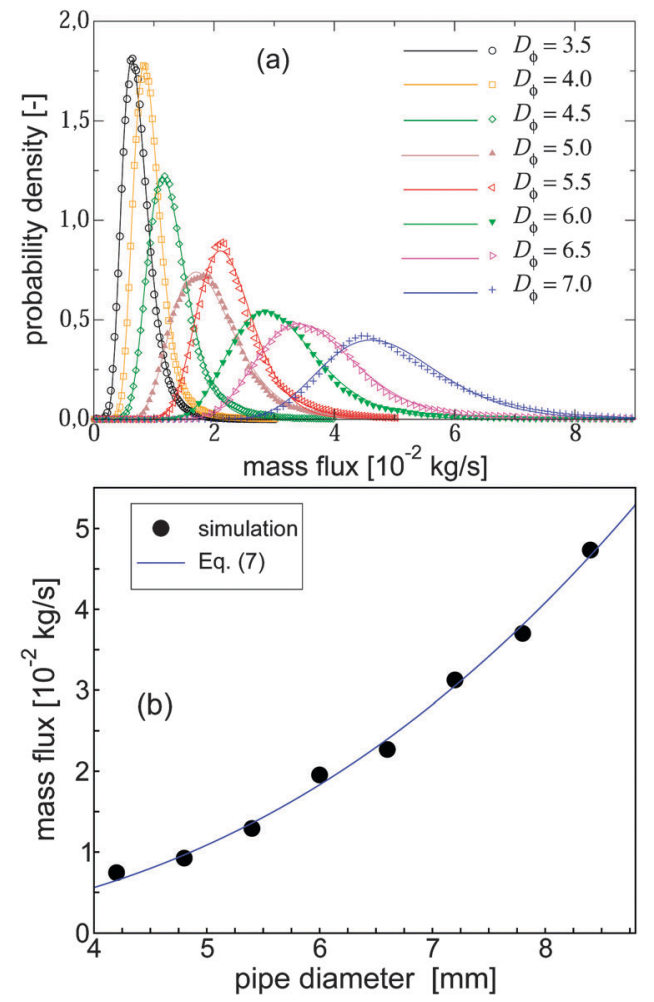

Fig. 2 Dependence of the mass flux on the pipe to particle diameter ratio $D_{\phi}$. The particle diameter is $d=1.2 \mathrm{~mm}$. (a) Probability density distribution of the mass flux for different values of $D_{\phi}$. Symbols denote simulation results, lines denote fits to the data using lognormal distributions; (b) expectation value of the mass flux as a function of the pipe diameter $D$. Circles denote simulation results, the solid line corresponds to the best fit to these data using eqn (7), which gives $A \approx 1.09$ and $k \approx 0.59$, with correlation coefficient $R^{2} \approx 0.998$.

flux density, with $\left\langle v_{z}\right\rangle$ denoting the average axial particle velocity. A scaling between $\left\langle v_{z}\right\rangle$ and the pipe diameter $D$ can be obtained by noting that the average flow rate is dictated by the behavior of grains within the plug zones. ${ }^{37}$ Each plug zone is associated with the formation of an unstable arch, the height of which should scale with $D$. Particles falling after the break of an arch can accelerate freely due to the action of gravity thus leading to a scaling of $\left\langle v_{z}\right\rangle$ with $\sqrt{D} .^{37}$ Therefore, a scaling of $Q_{0}$ with $D^{5 / 2}$ is expected. This scaling indeed governs the mass flux of a granular material flowing out a silo through an orifice of diameter $D$, which follows the well-known Beverloo equation, ${ }^{20,37-42}$

$$
Q_{0}=A \rho_{\mathrm{p}} \sqrt{g} \cdot(D-k d)^{2.5}
$$

where the coefficients $A$ and $k$ must be determined from the fit to the data. Such a scaling captures well the dependence of the mass flux on the pipe diameter observed in our simulations - the best fit to the simulation data is denoted by the continuous line in Fig. $2 \mathrm{~b}$.

It is important to remark that, in the regime of small pipe to particle diameter ratios $D_{\phi}$ investigated here, the distribution of the flow along the pipe may be strongly affected by geometric effects as the number of possible arrangements of the particles along the cross section of the pipe affects the probability of the formation of a stable arch (see Section 3.2). Such geometrical effects may influence the width of the probability density distribution curves in Fig. 2a as the value of $D_{\phi}$ is changed-for instance, we see that for $D_{\phi}=5.5$ the distribution is narrower than for $D_{\phi}=6.0$ and 5.0. However, we find that the mass flux increases monotonically with $D_{\phi}$ as seen in Fig. 2 b.

\subsection{Jamming regime}

The flow characteristics described above, with a constant average particle velocity and the formation of density waves along the pipe, persist over the entire simulation time, which was larger than $1000 \mathrm{~s}$ (real time of the physical system), for all values of $D_{\phi}>3$. In agreement with previous studies, ${ }^{43,44}$ we find that, for $D_{\phi} \lesssim 3$, jamming occurs thus leading to complete blockage of the granular flow. As an example of the flow in the jamming regime, the spatio-temporal diagram of the packing fraction for $D_{\phi}=2.5$ is shown in Fig. 3a. As we can see in this figure, after about $1.8 \mathrm{~s}$, a large, stable plug is formed, which corresponds to the dark horizontal lines in the spatio-temporal diagram. Indeed, this plug does not break up with the impact of the smaller particle groups that fall onto it. In other words, the frictional forces that yield the archs leading to plug formation ${ }^{3}$ are strong enough to sustain the downward pressure on the granular column.

Our simulations show that, although the critical $D_{\phi} \approx 3.0$ below which complete blockage occurs is robust with respect to the filling volume of the particles relative to the pipe volume, $V_{\phi}$, the time needed for the blockage to occur depends on this parameter. To quantify this dependence, we perform 100 numerical experiments for each value of $V_{\phi}$ using $D_{\phi}=2.5$ which is within the jamming regime. Fig. $3 \mathrm{~b}$ shows the resulting normalized cumulative distribution of the number of jammed simulations, $n_{\text {jam }}$, as a function of time.

We see that $n_{\text {jam }}$ is shifted to the left as $V_{\phi}$ increases, which means that, statistically, the flow jams the earlier the larger $V_{\phi}$. In order to collapse all curves, we first calculate the time $t_{0}$ at which $n_{\text {jam }}$ becomes larger than $0.1 \%$. This time is shown as a function of $V_{\phi}$ in the upper inset of Fig. 3c (circles). A fit to the data using $t_{0}=a_{0} V_{\phi}{ }^{-2}$, denoted by the continuous line, gives $a_{0} \approx 0.012 \mathrm{~s}$. The main plot of Fig. $3 \mathrm{c}$ shows $n_{\mathrm{jam}}$ as a function of $\left(t-t_{0}\right) / \tau$, where $\tau$ is the time required for $50 \%$ of the simulations with a given $V_{\phi}$ to jam. As we can see in the lower inset of Fig. 3c, this characteristic time $(\tau \approx 1.6 \mathrm{~s})$ is nearly independent of $V_{\phi}$.

These results can be understood by noting that the jamming probability increases with the probability that a stable arch along the cross section of the pipe is formed (and thus with $V_{\phi}$ ). This probability further depends on $D_{\phi}$, which controls the number of possible configurations of particle arrangements along the cross-section of the pipe. For a constant $V_{\phi}=0.175$, we compute $n_{\text {jam }}$ for different values of $D_{\phi}=2.5,2.6,2.7,2.8,2.9$ and 3.0 (see Fig. 4a). We see that for $D_{\phi}$ within the range $2.5 \leq D_{\phi} \leq 2.9$ the flow jams earlier the smaller $D_{\phi}$, whereas this trend of $n_{\text {jam }}$ with $D_{\phi}$ is not obeyed by the curve 

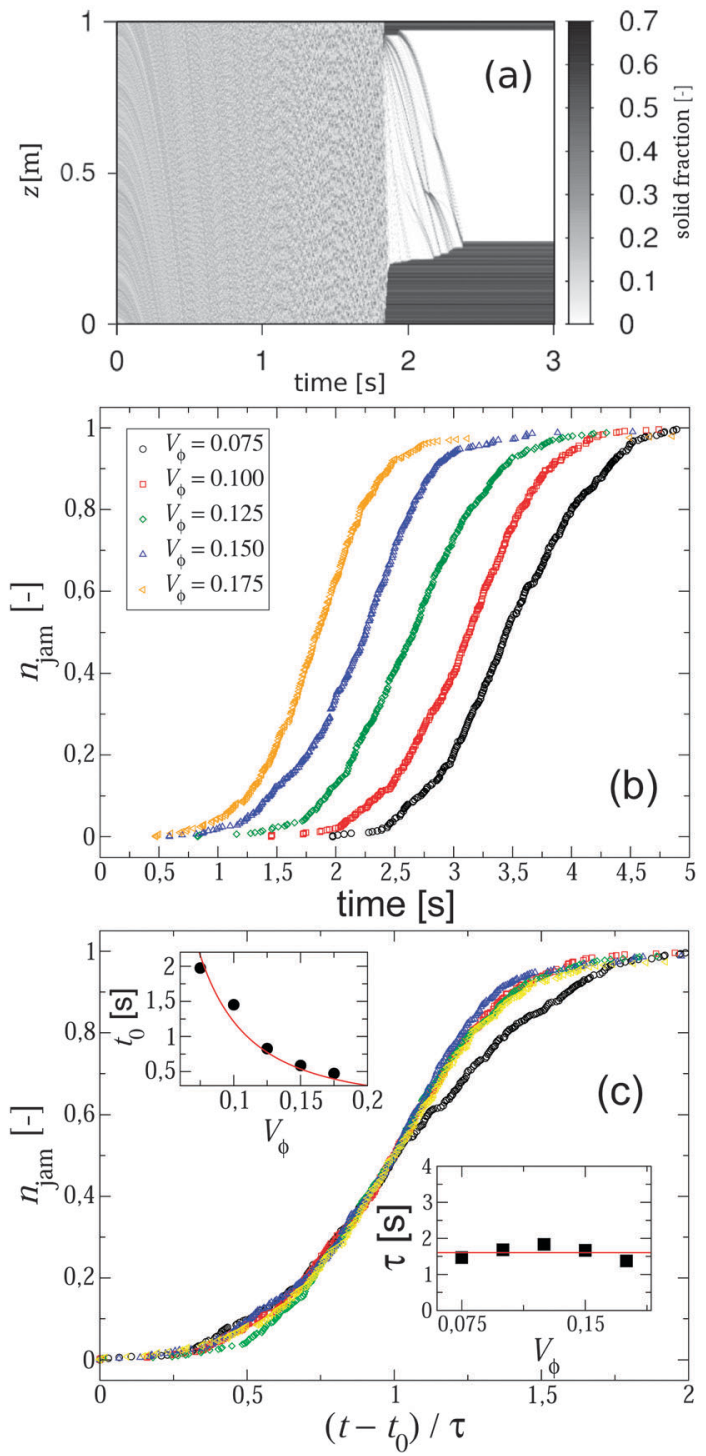

Fig. 3 (a) Spatio-temporal evolution of the solid fraction along the pipe for $D_{\phi}=2.5$ (jamming regime) and $V_{\phi}=0.175$. (b) Cumulative distribution of the number $n_{\mathrm{jam}}$ of jammed simulations with $D_{\phi}=2.5$ (jamming regime) as a function of time for different values of $V_{\phi}$; (c) $n_{\mathrm{jam}}$ as a function of the rescaled time $\left(t-t_{0}\right) / \tau$, where $t_{0}$ and $\tau$ are the times at which $n_{\text {jam }}$ is equal to $0.1 \%$ and $50 \%$, respectively. The continuous lines in the upper and lower insets denote fits to the simulation data using $t_{0}=a_{0} V_{\phi}^{-2}$ and $\tau=a_{\tau}$, which give $a_{0} \approx 0.012 \mathrm{~s}$, with correlation coefficient $R^{2} \approx 0.98$, and $a_{\tau} \approx 1.6 \mathrm{~s}$.

corresponding to $D_{\phi}=3.0$. The latter curve shows the largest jamming times among all $D_{\phi}$ as shown in Fig. 4 . To understand these results we consider the two-dimensional arrangements depicted in Fig. 4b-d. For $D_{\phi}=2.9$ (Fig. 4c) the probability of stable arch formation is larger than for $D_{\phi}=2.5$ (Fig. 4b), because in the former there is a higher probability to obtain a particle chain with a larger inter-particle contact area and thus a larger tangential force counter-balancing the particle weight. However, a linear arrangement of the particles parallel to the cross-section of the pipe can be obtained for $D_{\phi}=3.0$ (Fig. $4 \mathrm{~d}$ ), which dramatically decreases the probability of stable arch formation. Of course a much larger number of configurations
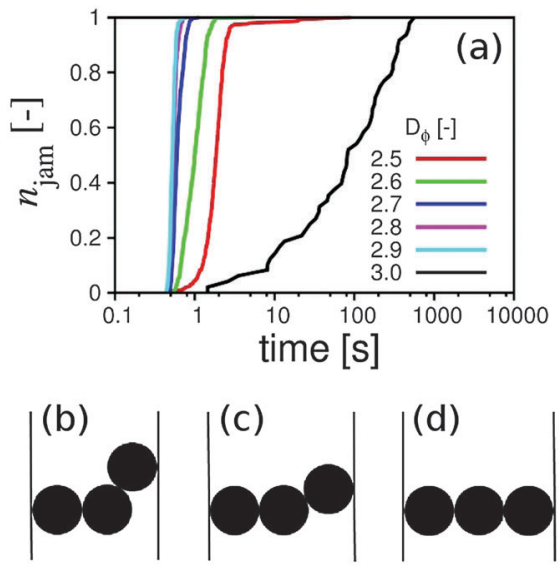

Fig. 4 (a) Cumulative distribution $n_{\text {jam }}$ as a function of time for different values of $D_{\phi}$ and for constant $V_{\phi}=0.175$. Note that the time is in the logarithmic scale; (b)-(d) show two-dimensional arrangements of particles along the cross section of the pipe with diameter ratios $D_{\phi}=2.5,2.9$ and 3.0, respectively.

is possible considering $D_{\phi}>3.0$ and three dimensional arrangements. However, although we indeed did not observe complete flow blockage for $D_{\phi}>3.0$, the flow in this regime is intermittent and characterized by density waves and intermittent transport as discussed in Section 3.1 and demonstrated previously. ${ }^{3,4}$

Therefore, in the following our aim is to develop a method to homogenize the flow thus avoiding the formation of density waves that lead to jamming in granular pipe flows.

\subsection{Flow homogenization by means of helical inner-wall texture}

Experiments aiming at reducing erosion damage from slurries in pipeline bends ${ }^{45}$ showed that pipes which encourage swirl can get particles into suspension at lower pumping power and pressure drop than a round duct. Inspired by this observation, we investigate the vertical flow of a granular material down a pipe of circular cross-section that has a helical inner-wall texture as depicted in Fig. 5a. This texture is constituted of small beads of diameter $d_{\mathrm{s}}=D / 10$, which are fixed to the innerwall of the pipe and have the material properties listed in Table 1. Each constituent bead is fixed to the wall at its center such that half hemisphere of each bead is within the inner volume of the pipe. The diameter of the pipe in the presence of the helix is adjusted such that the total volume within the pipe is the same as in the simulations without the texture elements.

Fig. 5b shows the spatio-temporal diagram of the packing fraction for the flow within a pipe with $D_{\phi}=2.5$ and using the same parameters as in the simulation of Fig. 3a. As we can see jamming does not occur in such a pipe in the presence of the helical texture. A steady downward flow of the granular material is observed, whereas the particles are more homogeneously distributed throughout the pipe compared to the simulations without the helical texture. Such improvement is observed without regard of the average radial velocity of the particles. Indeed, in Fig. $6 \mathrm{a}$ and $\mathrm{b}$ we show the spatio-temporal diagrams 


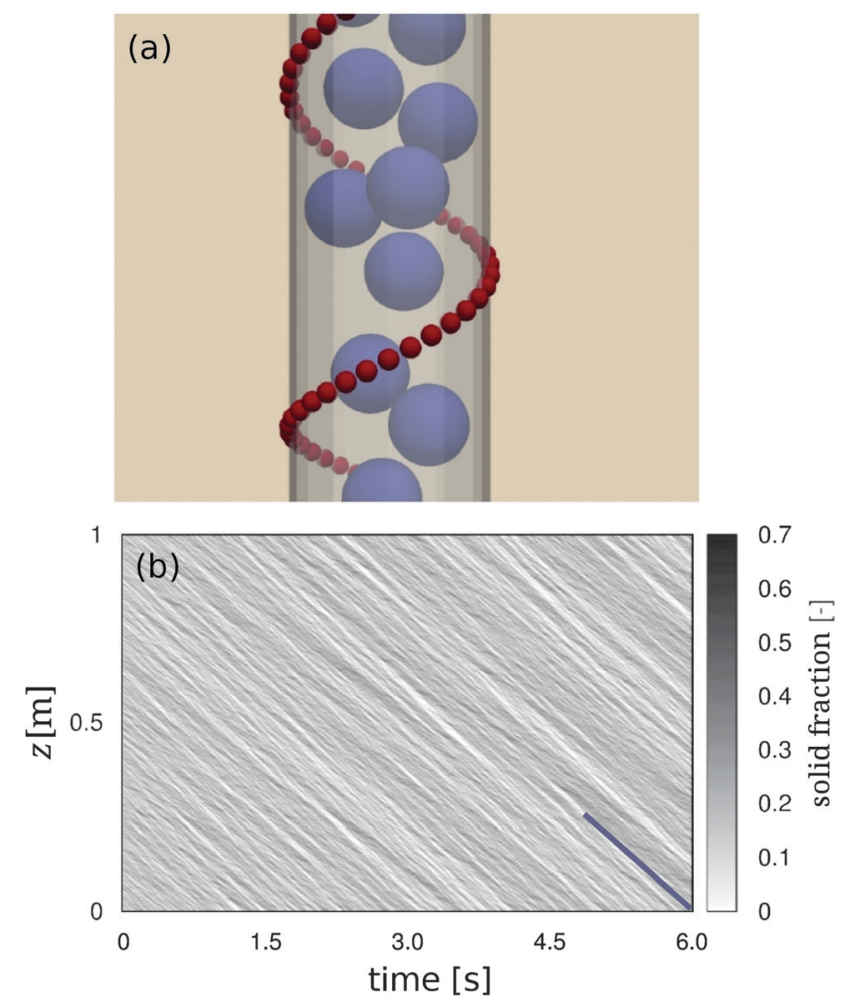

Fig. 5 (a) Flow of granular particles (blue) through a vertical pipe with $D_{\phi}=2.5$ to which the helical inner-wall texture (constituted by the red beads) is applied. The initial maximal radial velocity is $v_{\mathrm{r}}^{\max }=0.01 \mathrm{~m} \mathrm{~s}^{-1}$ as in Fig. 3; (b) corresponding spatio-temporal diagram of the solid fraction along the axial position. The slope of the line at the lower right corner indicates the average axial velocity of the particles.

of the packing fraction without and with helix texture, respectively, for the same pipe diameter but for an order of magnitude larger initial radial velocity. We see that the thick dark lines in the diagram of Fig. 6a are absent from the simulation with the helix texture (Fig. 6b). The flow in the presence of the helix takes place through smaller particle groups rather than through large plugs as in the conventional duct. This result can be understood by noting that, as the particles collide with the beads fixed on the inner-wall, they are deflected to the center of the pipe, thus hindering the formation of archs. Moreover, collisions between particles and the beads of the helix reduce the average axial velocity of the particles, whereas an increase in the mean radial velocity is observed (cf. Fig. 6c and d). Therefore, by introducing the helical texture of Fig. 5a, jamming can be prevented at the cost of a lower rate of particle flux down the pipe.

We find that the average mass flux depends significantly on the wavelength of the helix, $\lambda$. To illustrate this dependence, the spatio-temporal plots of the solid fraction for different values of $\lambda$ obtained in a simulation with $D_{\phi}=3.5$ (which corresponds to the clogging regime described in Section 3.1) are shown in Fig. 7. We see that the thick lines associated with the plugs in Fig. 7a give place to an increasingly more homogeneous flow as $\lambda$ decreases ( $c f$. Fig. $7 \mathrm{~b}-\mathrm{d}$ ). From the slope of the lines in the different spatio-temporal diagrams, we also see that the average
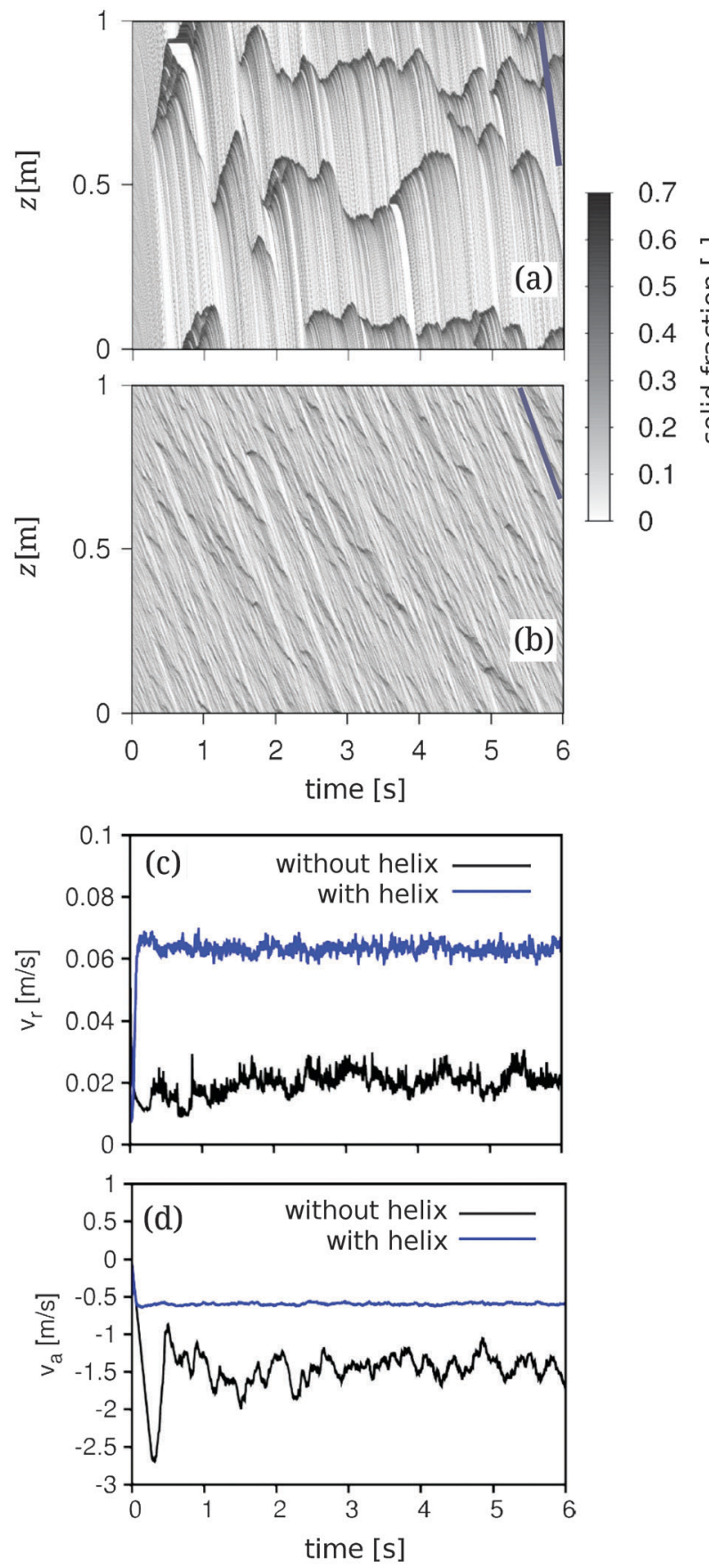

Fig. 6 Granular flow through a pipe with $D_{\phi}=2.5$ and $v_{\mathrm{r}}^{\max }=0.1085 \mathrm{~m} \mathrm{~s}^{-1}$. (a) Spatio-temporal plot of the solid fraction along the pipe without helix; (b) same plot for simulation with the helix; corresponding average values of the radial (c) and axial (d) velocities along the pipe, $\left\langle v_{r}\right\rangle$ and $\left\langle v_{a}\right\rangle$, respectively. The slope of the line at the upper right corner in each plot indicates the average axial velocity of the particles.

particle axial velocity increases with $\lambda$. This result can be further seen from Fig. 8, which shows the probability density functions $f\left(\dot{m}_{0}\right)$ of the mass flux $\dot{m}_{0}(c f$. Section 3.1), for different values of $\lambda$. As $\lambda$ decreases, $f\left(\dot{m}_{0}\right)$ approaches a Gaussian shape, that is, it becomes more symmetric, while the expectation value $Q=\left\langle\dot{m}_{0}\right\rangle$ of the mass flux also decreases. 

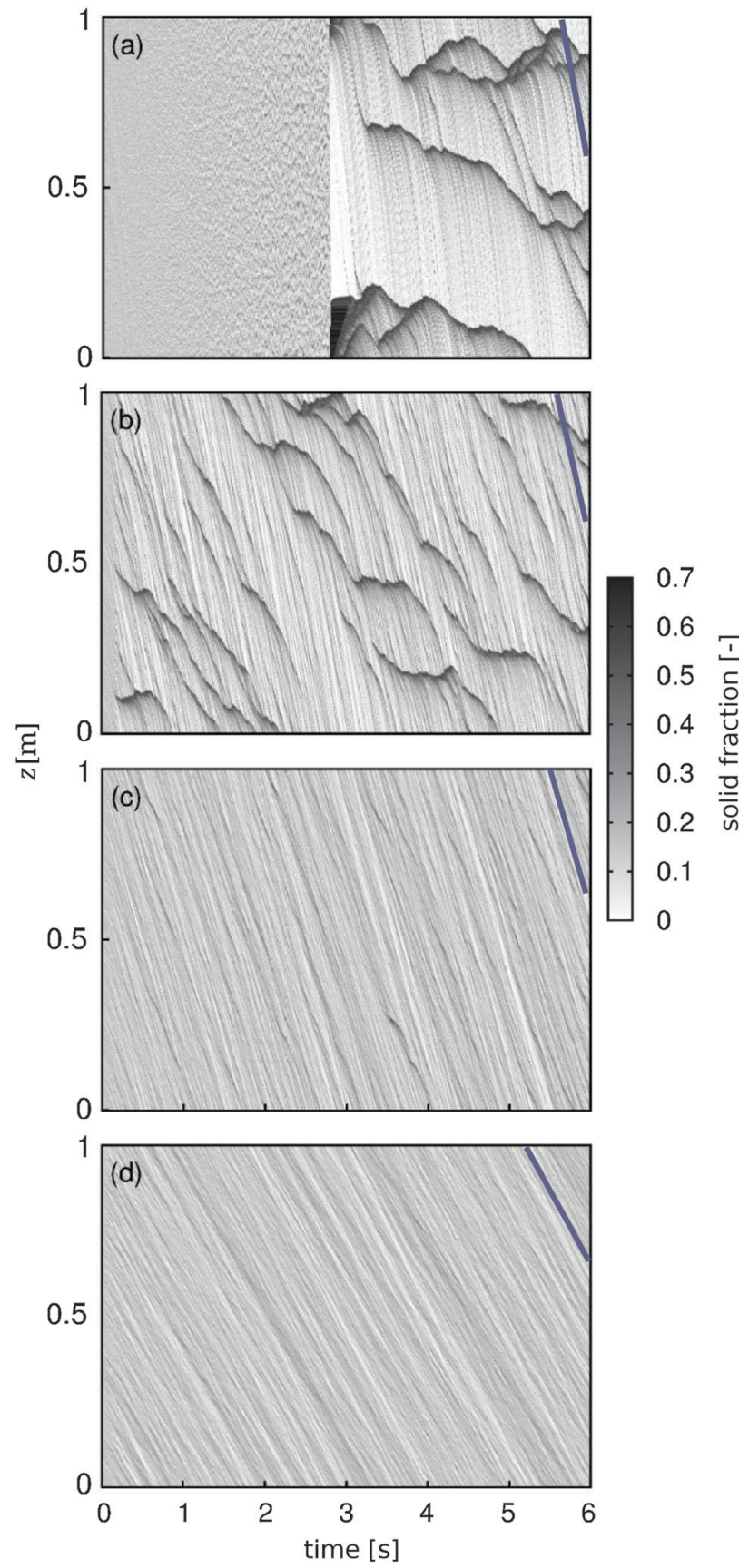

Fig. 7 (a) Spatio-temporal plots of the solid fraction along a pipe with $D_{\phi}=3.5$ and without helix; the subsequent figures show the same plots for simulations with a helix of wavelength $\lambda=1 \mathrm{~m}$ (b), $50 \mathrm{~mm}$ (c) and $12.5 \mathrm{~mm}$ (d). The slope of the line at the upper right corner in each plot indicates the average axial velocity of the particles.

In order to quantitatively describe the effect of the helix wavelength on the mass flux, we first note that in the steadystate, the gain in momentum of the particles due to gravity is fully compensated by the momentum loss due to collisions with the inner-wall of the pipe as well as with the other particles. In the presence of the helix, there is an additional contribution to the rate with which axial momentum is dissipated. This contribution, $\dot{p}_{\text {diss, helix }}$, is due to collisions of the

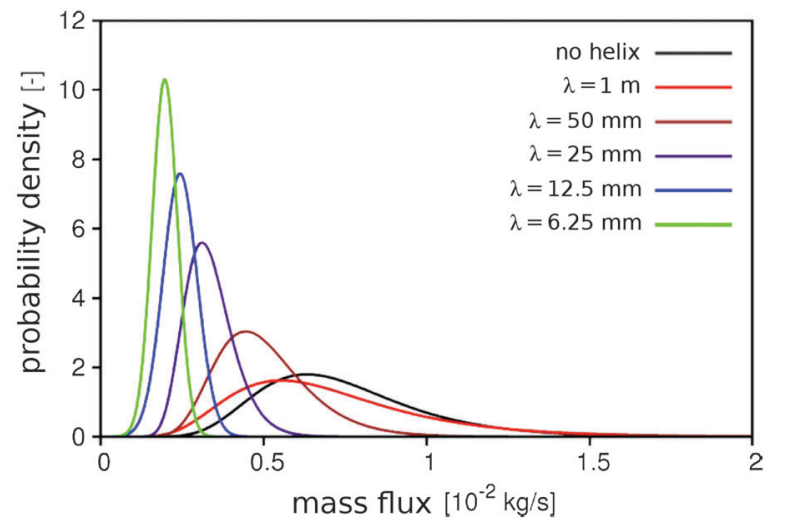

Fig. 8 Probability density distribution of the mass flux through a pipe with $D_{\phi}=3.5$ (that is, pipe diameter $D=4.2 \mathrm{~mm}$ ) for different values of the helix wavelength $\lambda$.

particles with the beads composing the helix. It is reflected by a relative decrease in the magnitude of the steady-state axial velocity of the particles, compared to the value without helix, as depicted in the example of Fig. 6d. Therefore, the steadystate flux $Q$ in the presence of the helix is related to the flux $Q_{0}$ without helix through the expression

$$
Q=Q_{0}-Q_{\text {diss,helix }}
$$

where $Q_{\text {diss, helix }}$ is the amount by which the steady-state axial mass flux is reduced when the helical texture is present.

In the small pipe to particle diameter ratio investigated here, it is reasonable to assume that the average mass flux in the axial direction is proportional to the average axial momentum of the particles, and that $Q_{\text {diss,helix }}$ is nearly proportional to $\dot{p}_{\text {diss,helix }}$. As a result of particle-helix collisions along the pipe, the particles are deflated to the central axis of the pipe which is why there is an increase in the average radial momentum when the helix is present (see Fig. 6c). However, we note that due to symmetry, for any radial direction, the contribution of the collisions to increase the radial momentum at the upper half of one helix wavelength is the same as at the lower half. For a given pipe diameter $D$ and a helix wavelength $\lambda$ made up of $n_{\mathrm{s}}$ beads, the collisions between particles with $n_{\mathrm{s}} / 2$ beads from the upper and lower hemispheres (half-wavelengths) deflate the particles in opposite directions. We thus need to calculate the rate with each the particles gain radial momentum due to collisions with one single hemisphere. The total rate is then twice the contribution from one half-hemisphere.

Fig. 9 presents both hemispheres unravelled from the innerwall as two right triangles, each with legs $D$ and $\lambda / 2$. Note that $\lambda / 2$ is the length corresponding to $n_{\mathrm{s}} / 2$ beads, which is the contribution of each hemisphere to increase the radial momentum along the cross section of the pipe. Moreover, the rate of axial momentum dissipation due to each hemisphere must scale with $p_{\perp}$, the component of the particles' average axial momentum $p_{0}$ perpendicular to the hypotenuse of each triangle. Each of both hypotenuses in Fig. 9 encompasses $n_{\mathrm{s}} / 2$ beads corresponding to one helix half-wavelength. Therefore, $\dot{p}_{\text {diss, helix }} \propto 2 p_{\perp}=2 p_{0} \sin \left[\Theta_{D, \lambda / 2}\right]$, where $\Theta_{D, \lambda / 2}=\arctan [\pi D /(\lambda / 2)]$ (see Fig. 9). Following our 


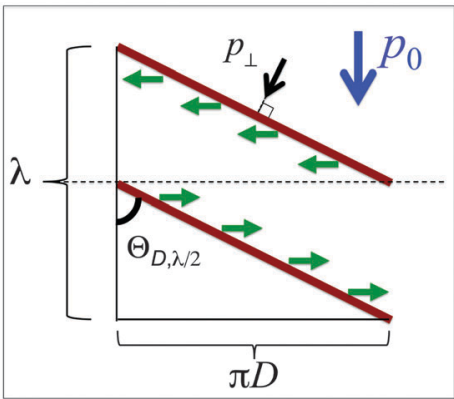

Fig. 9 Schematic diagram displaying the average axial momentum $p_{0}$ and the equivalent angle $\Theta_{D, \lambda / 2} \equiv \arctan [\pi D /(\lambda / 2)]$, which dictates the energy dissipation over one helix wavelength due to collisions with the $n_{\mathrm{s}} / 2$ beads composing one half-wavelength of the helix. The component $p_{\perp}=$ $p_{0} \sin [\arctan [\pi D /(\lambda / 2)]]$ is also indicated. The horizontal arrows along the helix indicate the direction from the helix to the central axis of the pipe.

assumption that $Q_{\text {diss,helix }}$ should be proportional to $\dot{p}_{\text {diss,helix }}$, we can write $Q_{\text {diss,helix }} \approx 2 b Q_{0} \sin [\arctan [\pi D /(\lambda / 2)]]=$ $2 b Q_{0} \cdot\left[2 \pi D / \sqrt{\lambda^{2}+(2 \pi D)^{2}}\right]$, where $b$ is a parameter that encodes information on the dissipative properties of the collisions. Thus, from eqn (8),

$$
\frac{Q}{Q_{0}}=1-B \cdot\left[\frac{2 \pi D}{\sqrt{\lambda^{2}+(2 \pi D)^{2}}}\right]
$$

where $Q_{0}$ is the flux without helix and the constant $B=2 b$ encodes information on energy dissipation due to collisions with the helix. Thus, the value of $B$ should depend on the material properties and particle diameter. To verify eqn (9), we compute the expectation value of the flux, $Q=\int q f_{\lambda}(q) \mathrm{d} q$ as a function of $\lambda$, where $f_{\lambda}(q)$ is the probability density function associated with the wavelength $\lambda$ as shown in Fig. 8. The result of this calculation is denoted by the symbols in the main plot of Fig. 10b, in which $Q$ appears rescaled with $Q_{0}$. The continuous line denotes the best fit using eqn (9), which gives $B \approx 0.74$. As we can see from this figure, the agreement between eqn (9) and the simulation results is excellent.

The inset of Fig. 10b shows the rescaled standard deviation of the flux, $\sigma / \sigma_{0}$, as a function of $1 / \lambda$, where $\sigma_{0}$ is the standard deviation obtained in the simulations without helix. The value of $\sigma / \sigma_{0}$ gives a measure of the homogeneity of the flux along the pipe-the smaller $\sigma$ the more homogeneous the flux. By fitting the simulation data using the equation

$$
\frac{\sigma}{\sigma_{0}}=1-C \cdot\left[\frac{2 \pi D}{\sqrt{\lambda^{2}+(2 \pi D)^{2}}}\right]
$$

we obtain $C \approx 0.9$. This fit is denoted by the continuous line in the inset of Fig. 10b. Therefore, both the flux and its standard deviation can be obtained from the geometric parameters of the helix using eqn (9) and (10), respectively.

Moreover, from eqn (9) we obtain a modification of the Beverloo equation (eqn (7)) for the flow of particulate materials in a vertical pipe of diameter $D$ in the presence of a helix of

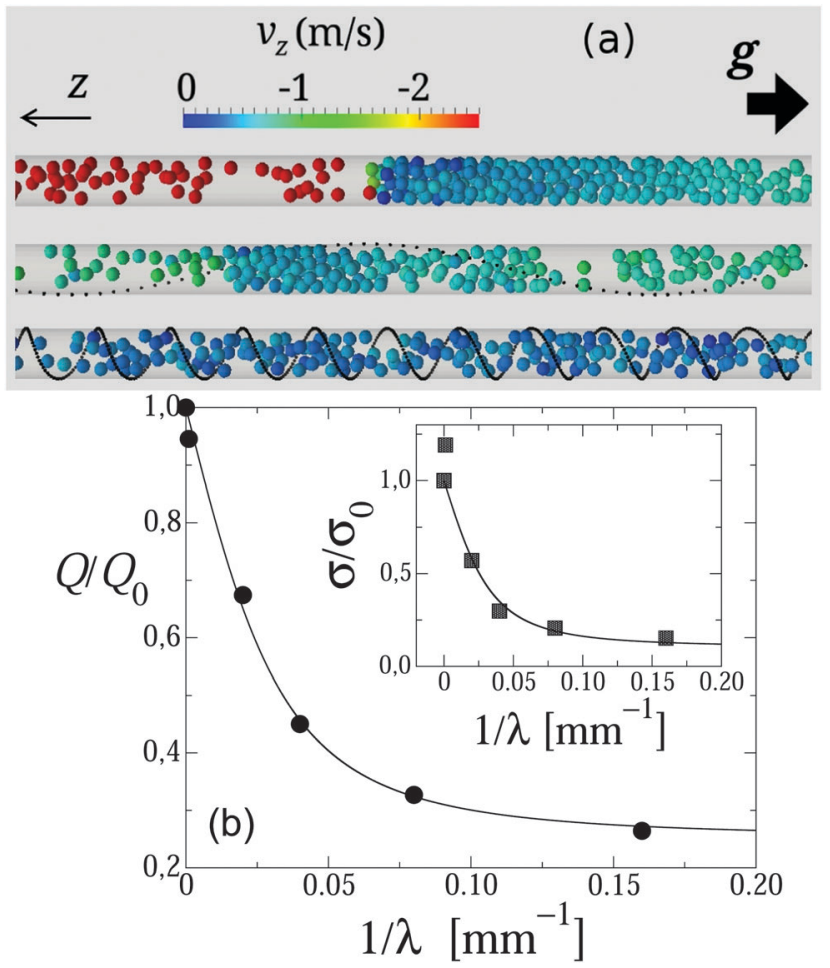

Fig. 10 (a) Snapshots of simulations without helix and with helix of wavelength values $\lambda=25 \mathrm{~mm}$ and $6.25 \mathrm{~mm}$ (from top to bottom). $D_{\phi}=$ 3.5 and in the figures a $70 \mathrm{~mm}$ long excerpt of the pipe is shown. The small particles composing the helix are coloured black and there are 44 of such particles per helix wavelength; (b) main plot: expectation value of the mass flux $Q$ as a function of $1 / \lambda$, where $\lambda$ is the helix wavelength and the parameters are the same as in Fig. 8. The flux is rescaled with $Q_{0} \approx$ $7.4 \times 10^{-3} \mathrm{~kg} \mathrm{~s}^{-1}$ which corresponds to the calculation with no helix, or equivalently $\lambda \rightarrow \infty$. The continuous line corresponds to the best fit to the simulation data using eqn (9), which gives $B \approx 0.75$. Inset: non-dimensional standard deviation $\sigma / \sigma_{0}$ as a function of $1 / \lambda$, where $\sigma_{0} \approx 2.5 \times 10^{-4} \mathrm{~kg} \mathrm{~s}^{-1}$ is the value of $\sigma$ with no helix. The continuous line denotes the best fit to eqn (10), which gives $C \approx 0.9$ with correlation coefficient $R^{2} \approx 0.983$.

wavelength $\lambda$. By replacing $Q_{0}$ in eqn (9) by the right-hand-side of eqn (7), we obtain,

$$
Q=A \rho_{\mathrm{p}} \sqrt{g}(D-k d)^{2.5} \cdot\left\{1-B \cdot\left[\frac{2 \pi D}{\sqrt{\lambda^{2}+(2 \pi D)^{2}}}\right]\right\}
$$

The circles in Fig. 11 show the mass flux as a function of the pipe diameter obtained for a constant helix wavelength $\lambda=$ $25 \mathrm{~mm}$. The corresponding fit to the simulation data using eqn (11) is shown by the continuous line and gives $A \approx 4.2, k \approx$ 1.13 and $B \approx 1.05$. We see that the agreement with the data is very good. However, obviously eqn (11) can only be valid for the regime of small pipe to particle diameter ratios investigated here. Indeed, the fit in Fig. 11 shows a maximum at $D_{\phi} \approx 6.5$, and predicts negative flux values for $D_{\phi} \gtrsim 10$. Moreover, as $\lambda \rightarrow 0$ the flux through the pipe must follow the original Beverloo equation with $Q_{0}$ corresponding to a pipe with no helix and diameter $D-d_{\mathrm{s}}$, where $d_{\mathrm{s}}$ is the size of the spheres constituting the helix. Further work is thus needed in order to elucidate the dependence of the coefficients $A, B, C$ and $k$ on the 


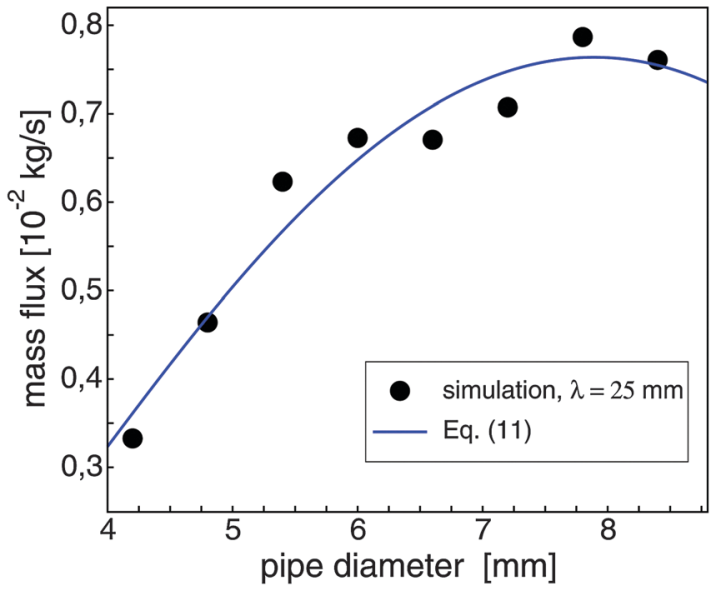

Fig. 11 Expectation value of the mass flux as a function of the pipe diameter $D$ for simulations using a helical inner-wall texture with $\lambda=$ $25 \mathrm{~mm}$. The particle size is $d=1.2 \mathrm{~mm}$ and the solid fraction $V_{\phi}=0.15$ is the same as in Fig. 2 (which considers simulations with no helix). Circles denote simulation results, and the continuous line denotes the best fit to these data using eqn (11), which gives $A \approx 4.2, k \approx 1.13$ and $B \approx 1.05$, with $R^{2} \approx 0.976$.

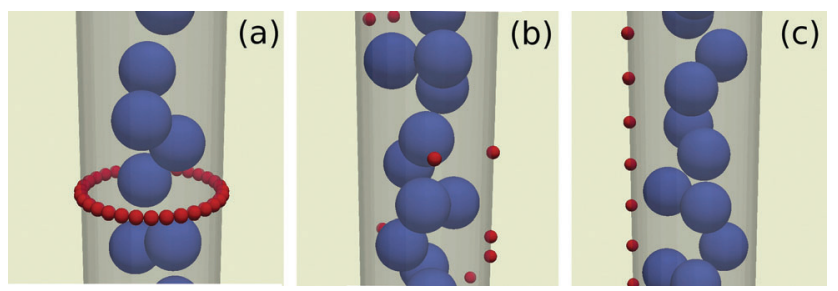

Fig. 12 Alternative texture geometries: (a) rings, (b) randomly distributed and (c) vertically aligned beads fixed on the inner-wall of the pipe. The number of particles constituting the different textures is the same as for a helical texture of wavelength $\lambda=23 \mathrm{~mm}$.

number of helix beads per wavelength $\lambda$, as well as on the frictional and dissipation properties of the particles.

Our results raise the question whether other inner-wall texture geometries have a similar effect as the helical one. We thus perform simulations using $V_{\phi}=0.15$ and $D_{\phi}=2.5$ with the helix geometry as well as with three further alternative textures (Fig. 12).

The first of these alternative geometries consists of rings disposed perpendicularly to the transport direction along the pipe axis and with spacing equal to $\lambda$. Each ring is made of the same type of beads that form the helix, and the number of beads in a ring is equal to the number of beads in one wavelength of the helix (Fig. 12a). The spatio-temporal diagrams obtained with the helix and with the rings are shown in Fig. 13a and b, respectively. We see that the texture made of rings does not favour steady flow in vertical pipes as does the helical one. Directly on top of each ring there occurs accumulation of particles and the formation of dense plugs which can lead to stable archs thus eventually causing blockage of the flow.

The second alternative geometry consists of disposing the constituent beads of the texture randomly over the entire
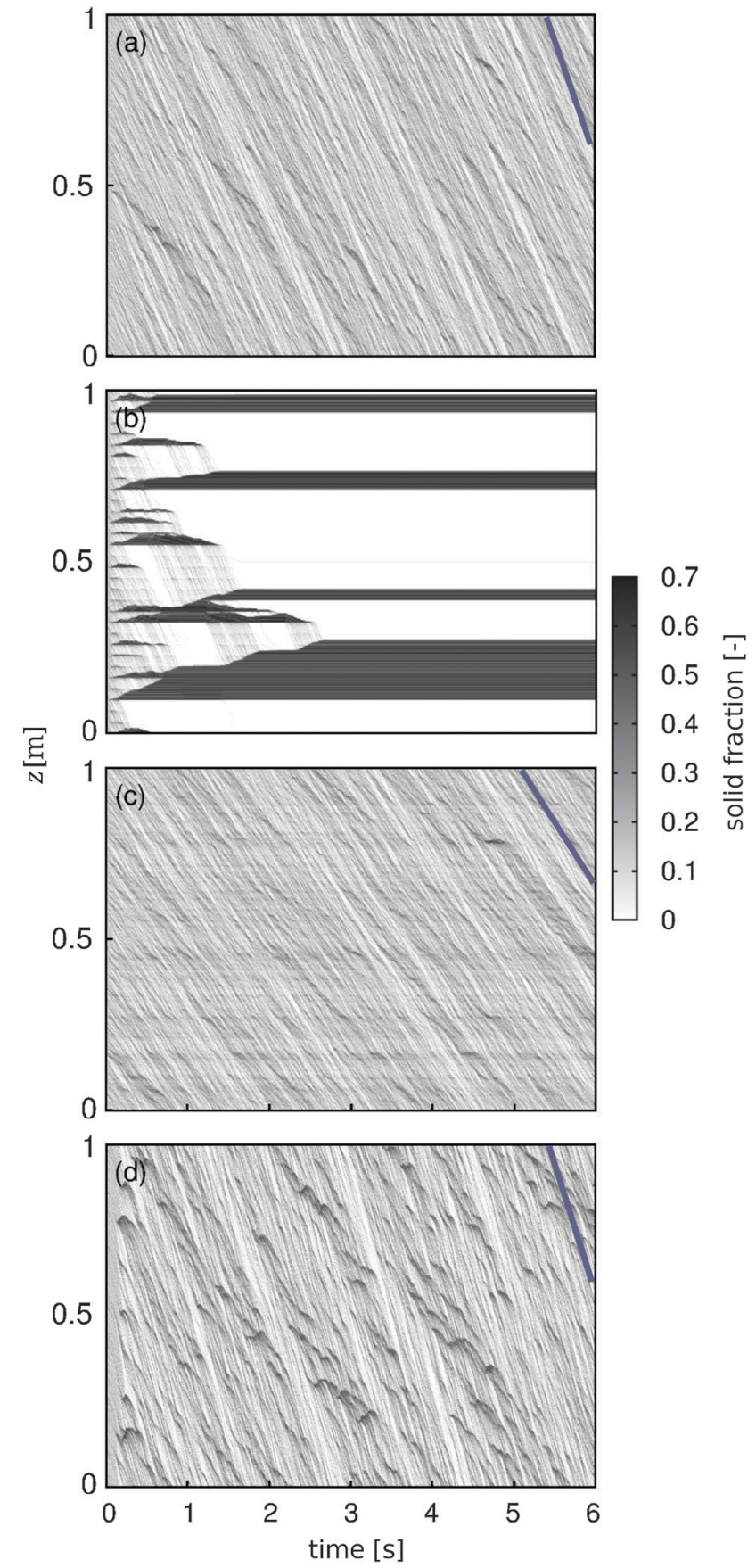

Fig. 13 Spatio-temporal plots of the solid fraction along the pipe for different types of inner-wall textures: (a) helix, (b) rings, (c) randomly distributed beads on the pipe inner-wall and (d) beads forming a single line that is parallel to the pipe axis. The slope of the line at the upper right corner in each plot indicates the average axial velocity of the particles.

inner-wall surface of the pipe (Fig. 12b). Hereby the same total number of constituent beads as in the helical texture is applied. By comparing the corresponding spatio-temporal diagram (Fig. 13c) with the one associated with the helix (Fig. 13a), we see that the latter texture geometry yields a larger particle flux - the particle axial velocity for the helix geometry is $0.71 \mathrm{~m} \mathrm{~s}^{-1}$, while for the random texture it is $0.39 \mathrm{~m} \mathrm{~s}^{-1}$. 
Finally, in the third alternative geometry the particles are arranged into a vertical line fixed on the inner-wall over the entire tube length (Fig. 12c). The spatio-temporal diagram of the simulation using such a texture, shown in Fig. 13d, displays the occurrence of dense plugs and inhomogeneous flow. Overall, the flow using the texture geometry in the form of a helix or using beads disposed randomly along the pipe is more homogeneous than the flow using the linear texture geometry.

Based on the results of our simulations, we conclude that the alternative textures are inferior to the helical inner-wall one since they lead either to inhomogeneous flow or to smaller mass flux values.

\section{Conclusions}

In conclusion, we have presented a method to obtain steady flows of granular materials through narrow pipes, which consists of applying a helical texture to the inner wall of the pipe. In the presence of such a texture, particle impingements are more homogeneouly distributed along the pipe, which substantially reduces the solid fraction fluctuations that are inherent to particulate flows through narrow channels. Our simulations show that, by tuning the wavelength of the helix, it is possible to achieve flows with prescribed transport characteristics. Specifically, we have shown that the mass flux in the presence of the helix can be predicted as a function of the pipe diameter and helix wavelength using an expression that is a modification of the well-known Beverloo equation with only one additional fit parameter. Excellent quantitative agreement between the values of mass flux predicted from our expression with the mass flux values obtained from simulation results was found.

To the best of our knowledge, there is no direct experimental evidence of the effect of a helical inner-wall texture on the flow of granular materials in vertical pipes, the present results could be compared to. However, as stated before, it was already shown experimentally that erosion damage from granulates in fluid flow through pipeline bends can be diminished by using helically-formed pipes, because such pipes, which encourage swirl, lead to a more homogeneous particle concentration. ${ }^{45}$

The helical inner-wall texture provides a means to homogenize the flow along the entire pipe without the need for energy input from any external source. In fact, previous methods to obtain steady granular flows were either based on energy input to the system, e.g. through electric fields or mechanical perturbations, ${ }^{1}$ or designed to prevent the blockage at the end of silos by adding an obstacle near the flow outlet. ${ }^{20}$ Our method has proven to be efficient to prevent the formation of density waves which are inherent to the flow of granular materials through pipes and occur without regard of the pipe to particle diameter ratio. We remark that the density waves in vertical pipe flows occur at low packing fraction values and that the necessary condition for jamming in such flows is the formation of a stable plug that does not break due to collisions of particles falling upon it thus leading to flow blockage and formation of a stable column. This mechanism is thus different from the shear-jamming mechanism where a dense granular system, which is already close to its highest packing fraction associated with the jammed stated, is driven to a shear-jammed state by application of a shear stress at constant density. ${ }^{46-49}$

We believe that application of the helical texture presented here could be used to enhance not only gravity-driven pipe flows but also fluid-driven particle transport both through vertical and horizontal pipes, ${ }^{18,50}$ which remain to be investigated in the future. It would be thus interesting to perform experiments on vertical or horizontal pipe flows that include a helical inner-wall texture to verify the predictions from our numerical simulations.

\section{Acknowledgements}

We thank Patric Müller, Dan Serero, Severin Strobl and Nikola Topic for discussions. We thank the German Research Foundation (DFG) for funding through the Cluster of Excellence "Engineering of Advanced Materials" and the Collaborative Research Center SFB814 (Additive Manufacturing). We gratefully acknowledge the computing time granted by the John von Neumann Institute for Computing (NIC) and provided on the supercomputer JUROPA at Jülich Supercomputing Centre (JSC).

\section{References}

1 C. Liu, P. Wu and L. Wang, Soft Matter, 2013, 9, 4762-4766.

2 K. To, P. Y. Lai and H. K. Pak, Phys. Rev. Lett., 2001, 86, 71-74.

3 T. Pöschel, J. Phys. I, 1994, 4, 499-506.

4 T. Riethmüller, L. Schimansky-Geier, D. Rosenkranz and T. Pöschel, J. Stat. Phys., 1997, 86, 421-430.

5 T. Le Pennec, M. Ammi, J. C. Messager, B. Truffin, D. Bideau and J. Garnier, Powder Technol., 1995, 85, 279-281.

6 O. Moriyama, N. Kuroiwa, M. Matsushita and H. Hayakawa, Phys. Rev. Lett., 1998, 80, 2833-2836.

7 J. L. Aider, N. Sommier, T. Raafat and J. P. Hulin, Phys. Rev. E: Stat. Phys., Plasmas, Fluids, Relat. Interdiscip. Top., 1999, 59, 778-786.

8 Y. Bertho, F. Giorgiutti-Dauphiné, T. Raafat, E. J. Hinch, H. J. Herrmann and J. P. Hulin, J. Fluid Mech., 2002, 459, 317-345.

9 Y. Bertho, F. Giorgiutti-Dauphiné and J. P. Hulin, Phys. Fluids, 2003, 15, 3358-3369.

10 O. Moriyama, N. Kuroiwa, S. Tateda, T. Arai, A. Awazu, Y. Yamazaki and M. Matsushita, Prog. Theor. Phys. Suppl., 2003, 150, 136-146.

11 R. Gudhe, R. C. Yalamanchili and M. Massoudi, Int. J. NonLinear Mech., 1994, 29, 1-12.

12 J. Lee and M. Leibig, J. Phys. I, 1994, 4, 507-514.

13 G. Peng and H. J. Herrmann, Phys. Rev. E: Stat. Phys., Plasmas, Fluids, Relat. Interdiscip. Top., 1995, 51, 1745-1758.

14 A. Valance and T. Le Pennec, Eur. Phys. J. B, 1998, 5, 223-229. 
15 E. D. Liss, S. L. Conway and B. J. Glasser, Phys. Fluids, 2002, 14, 3309-3326.

16 E. D. Liss, S. L. Conway, J. A. Zega and B. J. Glasser, Pharm. Technol., 2004, 78-96.

17 I. Bratberg, F. Radjai and A. Hansen, Phys. Rev. E: Stat., Nonlinear, Soft Matter Phys., 2005, 71, 011301.

18 M. Strauss, S. McNamara, H. J. Herrmann, G. Niederreiter and K. Sommer, Powder Technol., 2006, 162, 16-26.

19 S. Å. Ellingsen, K. S. Gjerden, M. Grøva and A. Hansen, Phys. Rev. E: Stat., Nonlinear, Soft Matter Phys., 2010, 81, 061302.

20 I. Zuriguel, A. Janda, A. Garcimartn, C. Lozano, R. Arévalo and D. Maza, Phys. Rev. Lett., 2011, 107, 278001.

21 W. Chen, M. Hou, K. Lu, Z. Jiang and L. Lam, Phys. Rev. E: Stat., Nonlinear, Soft Matter Phys., 2001, 64, 061305.

22 M. L. Hunt, R. C. Weathers, A. T. Lee, C. E. Brennen and C. R. Wassgren, Phys. Fluids, 1999, 11, 68-75.

23 J. Schäfer, S. Dippel and D. E. Wolf, J. Phys. I, 1996, 6, 5-20.

24 T. Pöschel and T. Schwager, Computational Granular Dynamics, Springer, Heidelberg, 2005.

25 H. Kruggel-Emden, E. Simsek, S. Rickelt, S. Wirtz and V. Scherer, Powder Technol., 2007, 171, 157-173.

26 H. Kruggel-Emden, S. Wirtz and V. A. Scherer, Chem. Eng. Sci., 2008, 63, 1523-1541.

27 N. V. Brilliantov, F. Spahn, J.-M. Hertzsch and T. A. Pöschel, Phys. Rev. E: Stat. Phys., Plasmas, Fluids, Relat. Interdiscip. Top., 1996, 53, 5382-5392.

28 P. A. Cundall and O. D. L. Strack, Geotechnique, 1979, 29, 47-65.

29 C. Kloss, C. Goniva, A. Hager, S. Amberger and S. Pirker, Prog. Comput. Fluid Dyn., 2012, 12, 140-152, www.liggghts.com.

30 T. Schwager and T. Pöschel, Phys. Rev. E: Stat., Nonlinear, Soft Matter Phys., 2008, 78, 051304.

31 T. Schwager and T. Pöschel, Phys. Rev. E: Stat. Phys., Plasmas, Fluids, Relat. Interdiscip. Top., 1998, 57, 650-654.

32 R. Ramrez, T. Pöschel, N. V. Brilliantov and T. Schwager, Phys. Rev. E: Stat. Phys., Plasmas, Fluids, Relat. Interdiscip. Top., 1999, 60, 4465-4472.
33 P. Müller and T. Pöschel, Phys. Rev. E: Stat., Nonlinear, Soft Matter Phys., 2011, 84, 021302.

34 T. Schwager, V. Becker and T. Pöschel, Eur. Phys. J. E: Soft Matter Biol. Phys., 2008, 27, 107-114.

35 C. H. Rycroft, A. V. Orpe and A. Kudrolli, Phys. Rev. E: Stat., Nonlinear, Soft Matter Phys., 2009, 80, 031035.

36 L. E. Silbert, D. Ertas, G. S. Grest, T. C. Halsey, D. Levine and S. J. Plimpton, Phys. Rev. E: Stat., Nonlinear, Soft Matter Phys., 2001, 64, 051302.

37 C. Mankoc, A. Janda, R. Arévalo, J. M. Pastor, I. Zuriguel, A. Garcimartn and D. Maza, Granular Matter, 2007, 9, 407-414.

38 W. A. Beverloo, H. A. Leniger and J. van de Velde, Chem. Eng. Sci., 1961, 15, 260-269.

39 G. H. L. Hagen, Poggendorffs Ann. Phys. Chem., 1839, 46, 423-442.

40 R. M. Nedderman, U. Tüzün, S. B. Savage and G. T. Houlsby, Chem. Eng. Sci., 1982, 37, 1597-1609.

41 B. P. Tighe and M. Sperl, Granular Matter, 2007, 9, 141-144.

42 A. Janda, I. Zuriguel, J. Bienzobas, A. Garcimartín and D. Maza, AIP Conf. Proc., 2013, 1542, 710-713.

43 C. A. Shook and M. C. Roco, Slurry Flow: Principle and Practice, Butterworth-Heinemann, Stoneham, MA, 1991.

44 J. Hadjigeorgiou and J. F. Lessard, Int. J. Rock Mech. Min. Sci., 2007, 44, 820-834.

45 R. J. K. Wood, T. F. Jones, N. J. Miles and J. Ganeshalingam, Wear, 2001, 250, 770-778.

46 D. Bi, J. Zhang, B. Chakraborty and R. P. Behringer, Nature, 2011, 480, 355-358.

47 M. Pica Ciamarra, P. Richard, M. Schröter and B. P. Tighe, Soft Matter, 2012, 8, 9731.

48 M. S. van Deen, J. Simon, Z. Zeravcic, S. Dagois-Bohy, B. P. Tighe and M. van Hecke, Phys. Rev. E: Stat., Nonlinear, Soft Matter Phys., 2014, 90, 020202(R).

49 R. Arévalo and M. Pica Ciamarra, Soft Matter, 2014, 10, 2728.

50 E. W. C. Lim, C.-H. Wang and A. B. Yu, AIChE J., 2006, 52, 496-509. 